Rev Inv Vet Perú 2009; 20 (1): 139-141

\title{
Comunicación
}

\section{ANÁLISIS DE AFLATOXINA M EN LECHE FRESCA DE ESTABLOS LECHEROS DE AREQUIPA}

\author{
Analysis of Aflatoxin M in Raw Mitk of Dairy Farms in Arequipa
}

\author{
César Ortiz Z. ${ }^{1}$
}

\section{RESUMEN}

El presente estudio tuvo como objetivo evaluar la presencia de aflatoxinas $M_{1}$ en muestras de leche fresca de establos de la cuenca de Arequipa. Se colectaron muestras de 10 establos lecheros de las irrigaciones de Santa Rita, Majes, La Joya y Lluta en 2 periodos (verano e invierno) del año 2008. Los establos se clasificaron en intensivos y extensivos de acuerdo al sistema productivo empleado. No se obtuvo ninguna muestra de leche contaminada a aflatoxinas $\mathrm{M}_{1}$, siendo un indicativo de que la presencia de este metabolito en leche no constituye un problema en la zona de estudio.

Palabras clave: aflatoxina $\mathrm{M}_{1}$, leche, establo, Arequipa

\section{Abstract}

The aim of the present study was to evaluate the presence of aflatoxin $M_{1}$ in raw milk samples from dairy farms of Arequipa milkshed. Milk samples were collected from 10 dairy farms located in the irrigated area of Majes, Santa Rita, La Joya and Lluta districts in two seasons (summer and winter) of 2008. Milk farms were classified as intensive and semintensive according to their productive system. Zero positive milk samples were obtained during the study and therefore, it is concluded that aflatoxin $M_{1}$ is not a contaminant of fresh milk in the region.

Key words: aflatoxin $\mathrm{M}_{1}$, milk, dairy farms, Arequipa

\footnotetext{
${ }^{1}$ Departamento de Campo, Gloria S.A. E-mail: cortiz@gloria.com.pe
} 
Las aflatoxinas $\left(B_{1}, B_{2}, G_{1}\right.$ y $\left.G_{2}\right)$ conforman un grupo de micotoxinas producidas por diferentes especies de hongos del genero Aspergillus (Coulombe, 1993; Celik et al., 2005). Estas contaminan los cereales y forrajes, ya sea en el campo, al momento de la cosecha, en el almacenamiento o en el procesamiento de alimentos balanceados, cuando las condiciones son favorables para su desarrollo (Espindola, 2006; Boudra et al., 2007).

La aflatoxina $\mathrm{B}_{1}$ es considerada el componente más tóxico producido por los hongos (Battacone et al., 2005). Luego que un animal la ingiere, de 0.5 a $5 \%$ de la toxina ingerida es biotransformada a Aflatoxina $\mathrm{M}_{1}$ en el hígado (González et al., 2004), la cual es un metabolito tan tóxico como el contaminante original, y es posteriormente excretado en la leche. La cantidad de Aflatoxina M encontrada en la leche representa normalmente del 1 al $2 \%$ de la Aflatoxina $\mathrm{B}_{1}$ ingerida, llegando a encontrarse hasta el $6 \%$ en vacas de alta producción (Boudra et al., 2007).

La aflatoxina $\mathrm{M}_{1}$ es clasificada por la Agencia Internacional de Investigaciones del Cáncer (IARC) como clase 2B, la cual incluye a las sustancias carcinogénicas para los humanos (Celik et al., 2005); por lo que su presencia en leche y derivados constituye un riesgo para la salud (Deveci y Sezgin, 2005). Esta toxina es motivo de control mediante legislación oficial en muchos países. La Unión Europea ha fijado el límite máximo permisible de $0.05 \mu \mathrm{g} / \mathrm{L}$ en leche fluida, siendo uno de los más bajos en el mundo (Souza et al., 1999).

A pesar del riesgo que involucra en la salud pública la contaminación de un alimento tan importante como la leche con este metabolito, poco o ningún estudio ha sido realizado en el país para evaluar su presencia en leche cruda. El objetivo del presente estudio fue evaluar la presencia de aflatoxina $\mathrm{M}_{1}$ en dos tipos de productores de leche (intensivo y extensivo) en dos momentos del año (verano e invierno) en la cuenca de Arequipa.
El estudio se desarrolló en las instalaciones de la Planta de Majes de la empresa Gloria S.A., ubicada en el Alto Siguas, distrito de Majes, provincia de Caylloma, Arequipa en febrero (verano) y julio (invierno) de 2008. Se trabajó con 2 tipos de productores, clasificados de acuerdo al sistema de crianza. Los productores de crianza intensivos estaban ubicados en las irrigaciones de Majes, Santa Rita y La Joya y se caracterizaron por tener hatos grandes (>250 vacas Holstein), altos niveles productivos, manejo tecnificado y alimentación en base a forraje conservado y concentrados. Los productores de crianza extensiva estaban ubicados en la irrigación de Lluta, y se caracterizaron por tener hatos pequeños ( $<5$ vacas Holstein), bajos niveles productivos, y alimentación al pastoreo, sin suplementación de alimentos balanceados.

Se realizaron dos muestreos de leche con un intervalo de cuatro días en cada periodo (verano e invierno) a cinco establos por tipo de productor. Las muestras $(200 \mathrm{ml}) \mathrm{se}$ colectaron directamente del tanque de enfriamiento de leche o de la tina de pesado en la planta de procesamiento en el caso de aquellos que entregaban la leche en porongos. El análisis de aflatoxinas se realizó el mismo día del muestreo y las muestras se mantuvieron refrigeradas en todo momento. Se empleó el test cualitativo Snap® Aflatoxin M1 (IDEXX Laboratories, EEUU), que tiene un nivel de sensibilidad de $0.50 \mathrm{ppb}$. Se siguió el protocolo de análisis recomendado por el fabricante. Se evaluó el efecto del tipo de productor y del periodo del año sobre la presencia de aflatoxinas en leche por medio del análisis de varianza, utilizando el software estadístico MINITAB® Release 14.12.0.

No se encontraron muestras positivas a aflatoxina M1 en ninguna de las 40 muestras analizadas. Estos resultados podrían esperarse considerando el bajo nivel de contaminación por aflatoxinas encontrado en el forraje y ensilado de maíz (Driehuis et al., 2008), insumo principal en la dieta de las vacas en crianza intensiva. Asimismo, el mayor o exclusivo uso de pasturas frescas durante el 
verano (Deveci y Sezgin, 2005) favorecen la ausencia de hongos. Además, el incremento constante del precio de los granos en el último año, principalmente del maíz, ha generado un desplazamiento de este insumo por otros en las dietas de las vacas (Staples, 2007), minimizando el riesgo de contaminación de la ración. Se concluye que la aflatoxina $\mathrm{M}_{1}$ no constituye un contaminante de la leche fresca procedente de la zona de estudio.

\section{Literatura Citada}

\section{Battacone G, Nudda A, Palomba M,} Pascale M, Nicolussi P, Pulina G. 2005. Transfer of aflatoxin $B_{1}$ from feed to milk and from milk to curd and whey in dairy sheep fed artificially contaminated concentrates. J Dairy Sci 88: 3063-3069.

2. Boudra H, Barnouin J, Dragacci S, Morgavi DP. 2007. Aflatoxin $M_{1}$ and ochratoxin A in raw bulk milk from french dairy herds. J Dairy Sci 90: 3197-3201.

3. Celik T, SarimehmetoÄlu B, Küplïlü Ö. 2005. Aflatoxin M1 contamination in pasteurized milk. Vet Arhiv 75(1): 57-65.

4. Coulombe R. 1993. Biological action of mycotoxins. J Dairy Sci 76: 880-891.
5. Deveci O, Sezgin E. 2005. Aflatoxin $M_{1}$ levels of skim milk powders produced in Turkey. J Food Drug Anal 13(2): 139-142.

6. Driehuis F, Spanjer MC, Scholten JM, Giffel MCT. 2008. Occurrence of mycotoxins in feedstuffs of dairy cows and estimation of total dietary intakes. J Dairy Sci 91: 4261-4271.

7. Espindola S. 2006. Micotoxinas y micotoxicosis en el ganado bovino lechero. Rev Chapingo Serie Zonas Aridas 5: 89-94.

8. Gonçãlez E, Pinto M, Manginelli S, Felicio J. 2004. Intoxicação de vacas leiteras por farelo de algodão naturalmente contaminado com aflatoxinas. Ciencia Rural 34(1): 171-174.

9. Souza S, Vargas E, Junqueira $R$. 1999. Eficiência de um Kit de ELISA na detecção e quantificação de aflatoxina $\mathrm{M}_{1} \mathrm{em}$ leite e investigação da ocorrência no estado de Minas Gerais. Ciênc Tecnol Aliment 19(3): 401-405.

10. Staples $\boldsymbol{C R}$. 2007. Feeding dairy cows when corn prices are high. [Internet], [2 September 2008]. Available in: http:/ /www.milkproduction.com/Library/Articles/feeding_dairy_cows_when_corn_ proces_are_high-htm 